\title{
An unusual presentation of nabothian cyst: a case report
}

\author{
Kanan A. Yelikar, Sonali S. Deshpande, Shubhangi F. Deshmukh*, Sanjay B. Pagare
}

Department of Obstetrics and Gynaecology, Govt. Medical College and hospital, Aurangabad, Maharashtra, India

Received: 20 July 2015

Revised: 10 August 2015

Accepted: 14 August 2015

*Correspondence:

Dr. Shubhangi F. Deshmukh

E-mail: kshubhangi709@gmail.com

Copyright: (C) the author(s), publisher and licensee Medip Academy. This is an open-access article distributed under the terms of the Creative Commons Attribution Non-Commercial License, which permits unrestricted non-commercial use, distribution, and reproduction in any medium, provided the original work is properly cited.

\begin{abstract}
Nabothian cysts are common gynecologic findings and rarely of clinical significance. They are not problematic unless they are sizeable and present secondary symptoms like in this case. Nabothian cysts are usually associated with chronic cervicitis, an inflammatory condition of cervix, and are harmless and usually disappear on their own. Here we report an interesting case of multiple large nabothian cysts presented with continuous, thin \& copious watery discharge, a distressing symptom, mimicking genitourinary fistula. This case is reported for the rarity of symptoms of continuous copious thin watery discharge per vaginum. Very rarely nabothian cyst needs hysterectomy as in our case.
\end{abstract}

Keywords: Nabothian cyst, Genitourinary fistula, Hysterectomy

\section{INTRODUCTION}

Nabothian cysts are common gynecologic findings and rarely of clinical significance. The squamocolumnar junction of the cervix is not a static tissue; the squamous epithelium of the ectocervix proliferates and covers the columnar epithelium of the endocervical glands. Nabothian cyst is formed when a cleft of columnar epithelium becomes covered with squamous cells and the columnar cells continue to secrete mucoid material. ${ }^{1}$ They can be solitary or multiple and their size shows considerable variations. The sizes of the cysts vary from microscopic to several centimetres. Nabothian cysts may occur by the inflammation and reparative processes of chronic cervicitis, following minor trauma or childbirth. ${ }^{2}$ Submucosal layer of the cervix is the most common location of these cysts, rarely they are seen deeply into the cervical wall. Here we report an interesting case of multiple large nabothian cysts presented with continuous, thin \& copious watery discharge, a distressing symptom, mimicking genitourinary fistula.

\section{CASE REPORT}

43 year old woman presented with continuous, clear \& copious watery discharge per vaginum since 7 years which disturbed her daily activities. Her menstrual pattern was normal. She gave history of undergoing two cesarean sections, last done 15 years back followed by tubal ligation 12 years back. She had consulted many gynaecologists and urologists over a period of 7 years, but there was no relief in her distressing symptoms. Her previous records showed a differential diagnosis of chronic cervicitis, vesicovaginal fistula, vesico-cervical fistula and vesicouterine fistula by various clinicians over the years. She was also extensively investigated over this period. However, there was no relief in her symptoms.

Now on per speculum examination continuous watery fluid was seen coming through cervical canal (Figure 1). On per vaginal examination uterus was bulky, anteverted, mobile \& fornices were free. At the end of clinical examination her provisional diagnosis was kept as tiny genitourinary fistula which was probably not visible. 
Microscopic \& biochemical examination of fluid was negative for urine. Transvaginal sonography was performed showing slightly bulky uterus \& multiple tiny heterogenous hypoechoeic cysts ranging from $1 \mathrm{~cm}$ to $3.5 \mathrm{~cm}$ in size, in endocervical canal suggestive of multiple nabothian cysts \& this findings was confirmed on MRI (Figure 2).

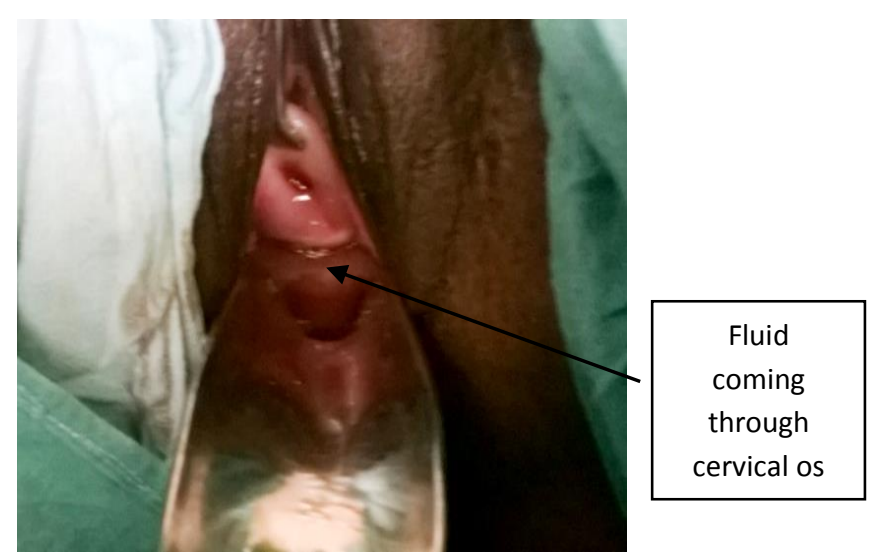

Figure 1: Per speculum examination showing thin watery fluid coming through os.

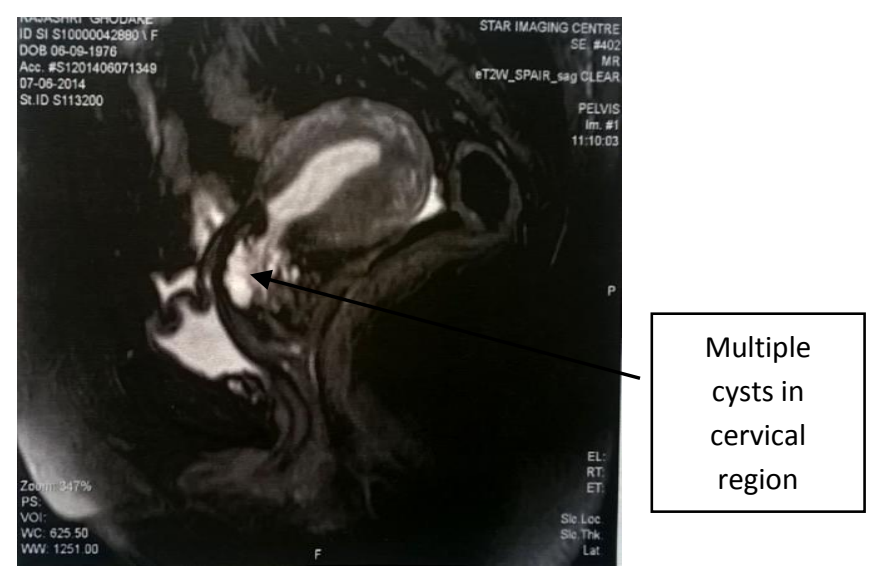

Figure 2: MRI showing multiple cysts in cervical region.

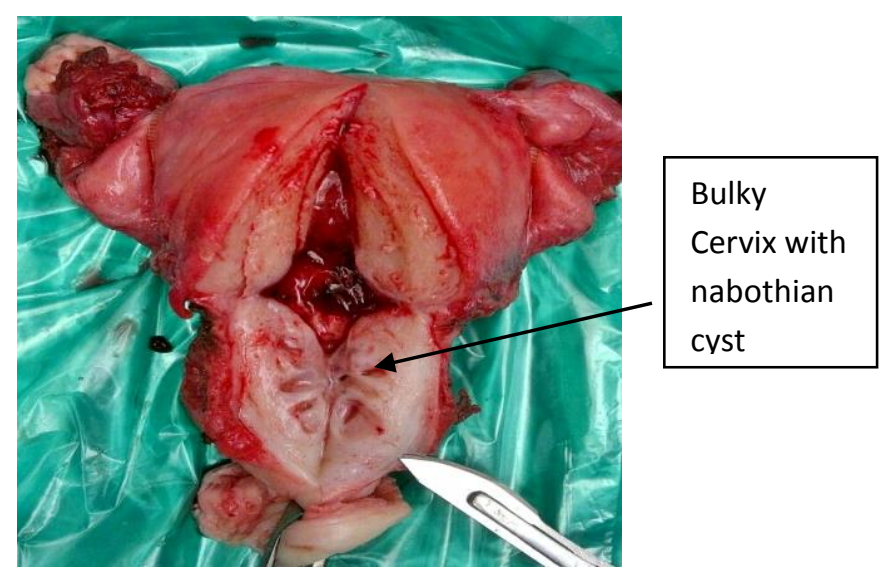

Figure 3: On cut section specimen showing multiple cysts in cervical region.
In view of her recurrent non-responding symptoms, total abdominal hysterectomy was performed, as a last resort. Cut section of uterine specimen showed multiple cysts of varying size filled with mucinous material (Figure 3). Her postoperative period was uneventful. The histopathology of specimen confirmed the diagnosis (Figure 4). She was followed up after surgery for 3 months. Finally her distressing symptoms were gone and she was smiling and satisfied.

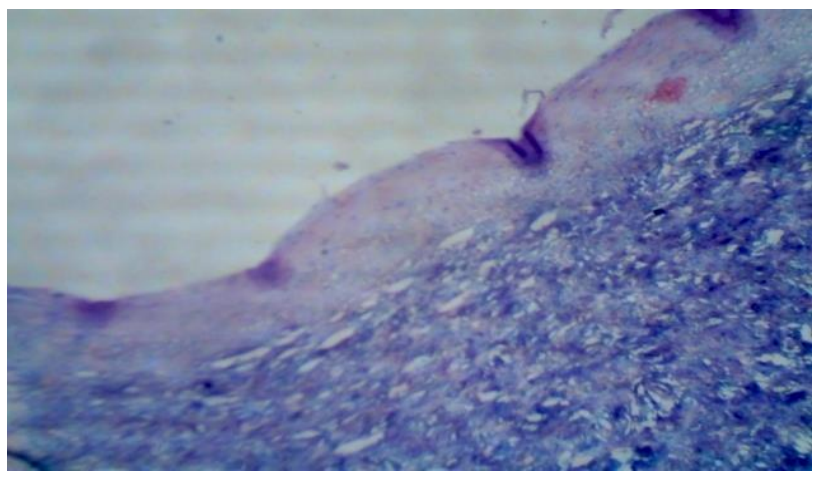

Figure 4: Histopathology slide showing nabothian cyst.

\section{DISCUSSION}

Nabothian cysts are the retention cysts in the cervix. Nabothian cysts are common, non neoplastic gynecologic disorder and rarely of any clinical significance. They are common and some reports suggest that they may see in up to $12 \%$ of routine pelvic MRI scans. ${ }^{3}$

Nabothian cysts appear as firm bumps on the surface of cervix which may be single or in groups. Nabothian cysts are usually associated with chronic cervicitis, an inflammatory condition of cervix, and are harmless and usually disappear on their own. They are not problematic unless they are sizeable and present secondary symptoms like in this case. In fact, most cases are detected accidentally, during pelvic examinations \& ultrasound. However, larger cysts can block the cervical opening and cause irregular bleeding as well as vaginal discharge. Some patients experience severe pain in the cervical region, especially during an intercourse. Intra-abdominal giant nabothian cyst presenting as lump in abdomen has been reported. ${ }^{3}$ Again a large nabothian cyst causing nulliparous prolapsed has also been reported. ${ }^{4}$

Transvaginal ultrasonography and magnetic resonance imaging are the most useful imaging modalities for cervical cystic lesions. ${ }^{5}$ Generally, nabothian cysts do not require any therapy. The therapy is recommended when a patient becomes symptomatic with pain or when the lesion character is not clear \& malignancy cannot be ruled out. When necessary cryocautery, electrocautery, cyst excision can be done. In rare cases hysterectomy may be needed. Temur et al reported a case of giant nabothian cyst compressing rectum which needed 
hysterectomy. ${ }^{3}$ Caglar et al reported hysterectomy for nabothian cyst presenting as chronic pelvic pain. ${ }^{5}$

\section{CONCLUSION}

Nabothian cyst is very common clinical entity. Nabothian cysts are mostly asymptomatic, but can lead to variable presentation making diagnosis difficult. This case is reported for the rarity of symptoms of continuous copious thin watery discharge per vaginum. Very rarely nabothian cyst needs hysterectomy as in our case. One should keep this condition in mind as a differential diagnosis in genito-urinary fistula.

Funding: No funding sources Conflict of interest: None declared Ethical approval: Not required

\section{REFERENCES}

1. Sosnovski V, Barenboim R, Cohen HI, Bornstein J. Complex nabothian cysts: a diagnostic dilemma. Arch Gynecol Obstet. 2009;279:759-61.

2. Kier R. non ovarian gynecologic cysts: MR imaging findings. AJR Am J Roentgenol. 1992;158:1265-9.

3. Temur I, Ulker K, Karaca M, Aydin A, Gurcu B. A giant cervical nabothian cyst compressing the rectum, differential diagnosis \& literature review. Clin Exp Obstet Gynecol. 2011;38:276-9.

4. A Nigam, D Choudhary, C Raghunandan. Large nabothian cyst: a rare cause of nulliparous prolapses. Case report Obstet Gynecol. 2012:1925-6.

5. Caglar Y, Asker ZO, Selda B, Dincer S, Ali C. Multiple \& large nabothian cysts: a case report. Cumhuriyet Med J. 2009;31:456-9.

Cite this article as: Yelikar KA, Deshpande SS,

Deshmukh SF, Pagare SB. An unusual presentation of nabothian cyst: a case report. Int J Reprod Contracept Obstet Gynecol 2015;4:1589-91. 